\title{
Prevalence and genotype distribution of HPV infection among 214,715 women from Southern China, 2012-2018: baseline measures prior to mass HPV vaccination
}

Li-pei Luo ${ }^{1}$, Ping He', Qiao-tu Liu', Yang-hua Jiang ${ }^{1}$, Yang-nan Zhang ${ }^{1}$, Qing-zhao Li', Qiu Li ${ }^{1}$, Sheng-tao Li', Fan Yang ${ }^{1}$, Hua Ling ${ }^{1}$, Xin-gui Dai ${ }^{1}$, Zhong-yu $\mathrm{Li}^{1,2^{*}}$ and Hong-liang Chen ${ }^{1,2^{*}}$ (D)

\begin{abstract}
Background: The epidemiology on the human papillomavirus (HPV) among females in Southern China is not wellestablished. Baseline data on the prevalence of HPV infection in China prior to mass prophylactic HPV vaccination would be useful. Thus, this study aims to determine the type-specific HPV prevalence and distribution among females from Southern China prior to mass HPV vaccination.

Methods: A retrospective cross-sectional study employing 214,715 women attending ChenZhou NO.1 People's Hospital for cervical screening during 2012-2018 was conducted prior to widespread HPV vaccination. HPV genotype was detected using nucleic acid molecular diversion hybridization tests. The overall prevalence, agespecific prevalence, type distribution, and annual trend were analyzed.

Results: The overall HPV prevalence was 18.71\% (95\% confidence interval [CI], 18.55-18.88\%) among Southern China females. During 2012-2018, the prevalence of HPV infection showed a downward tendency, from 21.63\% (95\% Cl, 21.07-22.20\%) in 2012 to $18.75 \%(95 \%$ Cl, 18.35-19.16\%) in 2018. Age-specific HPV distribution displayed a peak at young women aged less than 21 years $(33.11,95 \% \mathrm{Cl}, 31.13-35.15 \%), 20.07 \%$ (95\% Cl, 19.70-20.44\%) among women aged $21-30$ years, $17.29 \%(95 \% \mathrm{Cl}, 17.01-17.57 \%)$ among women aged $31-40$ years, $17.23 \%(95 \% \mathrm{Cl}, 16.95-$ $17.51 \%)$ among women aged $41-50$ years, $21.65 \%(95 \% \mathrm{Cl}, 21.11-22.20 \%)$ among women aged $51-60$ years, and $25.95 \%$ (95\% Cl, 24.86-27.07\%) among women aged over 60 years. Of the 21 subtypes identified, the top three prevalent high-risk HPV (HR-HPV) genotypes were HPV52 (5.12\%; 95\% Cl, 21.11-22.20\%), - 16 (2.96\%; 95\% Cl, 2.893.03\%), and - 58 (2.51\%; 95\% Cl, 2.44-2.58\%); the predominant low-risk HPV (LR-HPV) genotypes were HPV81 (1.86\%; $95 \% \mathrm{Cl}, 1.80-1.92 \%)$ and $-6(0.69 \% ; 95 \% \mathrm{Cl}, 0.66-0.73 \%)$ respectively. Incidence of HR-HPV only, LR-HPV only and mixed LR- and HR-HPV were 15.17, 2.07 and 1.47\% respectively. Besides, single HPV infection accounted for 77.30\% of all positive cases in this study.

(Continued on next page)
\end{abstract}

*Correspondence: Izhy1023@hotmail.com; chenhongliang2007@126.com

${ }^{1}$ The First School of Clinical Medicine, Southern Medical University, Chenzhou NO.1 People's Hospital, Chenzhou 423000, P.R. China

Full list of author information is available at the end of the article

(c) The Author(s). 2021 Open Access This article is licensed under a Creative Commons Attribution 4.0 International License, which permits use, sharing, adaptation, distribution and reproduction in any medium or format, as long as you give appropriate credit to the original author(s) and the source, provide a link to the Creative Commons licence, and indicate if changes were made. The images or other third party material in this article are included in the article's Creative Commons licence, unless indicated otherwise in a credit line to the material. If material is not included in the article's Creative Commons licence and your intended use is not permitted by statutory regulation or exceeds the permitted use, you will need to obtain permission directly from the copyright holder. To view a copy of this licence, visit http://creativecommons.org/licenses/by/4.0/ The Creative Commons Public Domain Dedication waiver (http://creativecommons.org/publicdomain/zero/1.0/) applies to the data made available in this article, unless otherwise stated in a credit line to the data. 
(Continued from previous page)

Conclusions: This study highlights 1) a high prevalence of HPV infection among females with a decreasing tendency towards 2012-2018, especially for young women under the age of 21 prior to mass HPV vaccination; 2) HPV52, - 16 and - 58 were the predominant HPV genotypes, suggesting potential use of HPV vaccine covering these HPV genotypes in Southern China.

Keywords: Human papillomavirus, High-risk HPV, Low-risk HPV, Prevalence, Genotype, Single HPV infection, Multiple HPV infection

\section{Background}

Human papillomavirus (HPV) is the leading cause of cervical cancer and responsible for about $91 \%$ of anal cancers, $75 \%$ of vaginal cancers and $70 \%$ oropharynx cancer [1]. It is estimated that there were over 528,000 new cervical cancer patients and 266,000 death worldwide recorded in 2012, among which $85 \%$ occurred in developing countries [2]. In China, 130,000 new cases of cervical cancer are diagnosed annually, especially in young women within the first few years after sexual debuts $[3,4]$. The morbidity and mortality of cervical cancer in developed countries has fallen over the last two decades because of an enhanced awareness of cervical cancer prevention and effective screening [5]. However, infection rate of HPV has fluctuated and not shown a downward trend, indicating a relatively heavy burden of HPV infection in China [6, 7].

HPV genotypes are linked to the degree of cervical lesions [6]. Infections with low-risk HPV (LR-HPV) types, such as HPV6 and-11, cause benign or low-grade changes in cervix cells, genital warts, and recurrent respiratory papillomatosis. Contrarily, high-risk HPV (HRHPV) type cause cervical, anal, and other genital cancers, which could be detected in $99 \%$ of cervical cancers-the second most common cancer in women worldwide. HPV16 and - 18 have been found to be the most pathogenic HR-HPV types, causing about $70 \%$ of cervical cancers worldwide [7-10]. Of note, most HPV infections are completely asymptomatic, resulting in a delay in diagnosis and follow-up treatment with disastrous consequences [11]. Moreover, HPV-infected individuals without early intervention will develop long-lasting HPV infections that put them at risk for cervical cancer, and increase the risk of sexual transmission to their partners, resulting in approximately estimated $\$ 1.7$ billion in direct medical costs annually in the United States [12].

The prevalence of HPV varies geographically widely, ranging from $6 \%$ in southeastern Asia to $32 \%$ in eastern Africa, and from 6.7 to $44.5 \%$ in China [13]. HPV screening and vaccine are considered being the most effective measures for preventing HPV infection. HPV vaccine has been clinically applied for more than 10 years [14], however, officially launched on July 2017 in mainland China and available mainly in the developed regions in China currently, such as Beijing, Shanghai, and Guangzhou. Besides, the HPV vaccine made in China was approved in 2020. Representative data on typespecific prevalence of HPV infection in China could provide a baseline estimation to the burden of HPV infection and could help guiding programs on HPV-based cervical cancer screening and strategies on vaccinebased HPV prevention. To date, the existing data about epidemiological characteristics of HPV infection in Southern China are still scantly, especially lacking large sample study on the genotype prevalence of HPV. Therefore, this paper was conducted to determine the prevalence and type distribution of HPV among 214,715 females in Southern China, including its trends from 2012 to 2018 and age-specific prevalence. Such data provide a baseline pre-vaccine population-based prevalence of HPV in Southern China, and help guiding models evaluating impact and cost-effectiveness by comparison between future vaccinated populations, and implementation of the prophylactic HPV vaccine according to the specific types' distribution in the region.

\section{Methods \\ Study population}

From January 2012 to December 2018, 214,715 females (age range from 19 to 83 years) were enrolled in this study. All participants were from gynecology department and physical examination center of Chenzhou NO.1 People's Hospital. They have got HPV tests for various reasons, including physical examination, vaginitis, urethritis, irregular vaginal bleeding, cervicitis, undiagnosed abdominal pain and genital warts. In addition, repeated samples from the same women were excluded.

\section{Cervical specimen collection and management}

All of the cervical samples were taken by the physician, but not self-sampling, using a $200 \mathrm{~mm}$ polyethylene Cervix brush device (Hybribio Corp, Guangdong) following the regular procedures for speculum examination. The samples were transferred to a sampling tube containing a transport medium (Hybribio Corp, Guangdong) and stored at $2-8{ }^{\circ} \mathrm{C}$ until HPV DNA extraction within a week. Cervical samples can be kept in a transport 
medium for 2 weeks at $4{ }^{\circ} \mathrm{C}$ according to the manufacturer's manual.

\section{HPV genotyping}

DNA was extracted by Cell Lysis Kit (Hybribio Corp, Guangdong) accompanying with the negative and positive quality control products throughout the whole process. HPV genotyping have been performed using HPV assay kit (Hybribio Corp, Guangdong) since 2012 with no modifications. This kit detected 21 HPV genotype via gene amplification technology and diversion hybridization. The low limit of detection (LOD) for HPV DNA is 500 copies/ml according to manufacturer's protocol. This assay uses HPV L1 consensus PCR primers (MY09/11) for the amplification and a human housekeeping gene $\beta$-globin as an endogenous internal control to ensure appropriate DNA purification, PCR reaction and specimen quality. PCR was carried out in $25 \mu \mathrm{l}$ reaction mixture in a thermal cycler, the cycling parameters of which were as follows: $20^{\circ} \mathrm{C}$ for $10 \mathrm{~min}$, $95^{\circ} \mathrm{C}$ for $9 \mathrm{~min}$, followed by 40 cycles $\left(20 \mathrm{~s}\right.$ at $95^{\circ} \mathrm{C}, 30 \mathrm{~s}$ at $55^{\circ} \mathrm{C}$ and $30 \mathrm{~s}$ at $72{ }^{\circ} \mathrm{C}$ ), with a final extension at $72^{\circ} \mathrm{C}$ for $5 \mathrm{~min}$. HPV types were then classified via a nylon membrane immobilized with 21 different type-specific probes. Quality controls of HPV genotyping including External Quality Assessment and Internal Quality Control were implemented throughout the study.

\section{Statistical analysis}

Analyses were conducted with SPSS version 19.0. Descriptive statistical analysis was performed on the distribution of HPV genotypes using indicators such as frequency and prevalence. The Chi-square test was used to test the differences between prevalence, genotypes and number of co-infections of HPV in different age groups and time groups.

\section{Ethical consideration}

The study was approved by the ethics Committee of Chenzhou NO.1 People's Hospital and conducted strictly in accordance with the Declaration of Helsinki, including the confidentiality and anonymity. No informed consent or other action on the part of the patients was required due to anonymous analyses of the data. All experiments were carried out in the lab certified by the National Center for Clinical Laboratories following the laboratory biosafety guidelines.

\section{Results}

\section{Overall prevalence and genotype distribution of HPV}

From 2010 to 2018, 214,715 eligible female participants were enrolled for final statistical analysis in this study. Among them, 40,168 cases (18.71\%; 95\% CI, 18.55$18.88 \%$ ) were positive for at least one HPV genotype
(Table 1). A general decline in prevalence was observed over the years from $21.63 \%(95 \% \mathrm{CI}, 21.07-22.20 \%)$ in 2012 to $18.75 \%$ (95\% CI, 18.35-19.16\%) in 2018. The prevalence of single and multiple HPV infections, as well as HPV genotypes except for HPV51, -39 and -53 also showed a similar decreasing trend (Fig. 1a, b).

HPV52 (5.12\%; 95\% CI, 5.03-5.21\%) was the most common genotype, followed by HPV16 (2.96\%; 95\% CI, 2.89-3.03\%) and - 58 (2.51\%; 95\% CI, 2.44-2.58\%), accounting for $76.36 \%$ of all detected HPV types (Table 1), which were also the top three prevalent genotypes among HR-HPV, while its counterpart LRHPV was HPV81, -6 and -11 , respectively (Table 1 and Fig. 2).

\section{Prevalence of high-risk, low-risk HPV by age and types} The overall HR-HPV and LR-HPV positive rates were 20.63\% (95\% Cl 20.46-21.80\%) and 3.67\% (95\% Cl 3.59$3.75 \%)$ respectively. HR-HPV infection was significantly higher among women with single infection (12.46\%) than those with multiple HPV infections $(8.17 \%, P<$ 0.001 ), and a similar result was also observed for LRHPV infection ( $2.0 \%$ vs. $1.67 \%, P<0.001$, Table 1$)$. When dividing the subjects into six age groups $(<21,21-30$, $31-40,41-50,51-60$ and $>60$ years), we found the highest prevalence of HPV infection was observed among females under 21 years old $(33.11 \%$; 95\% CI, $31.13-$ $35.15 \%$ ), while the lowest HPV prevalence occurred in women aged $41-50$ years (17.23\%; 95\% CI, 16.95$17.21 \%)$. There was a statistically significant difference in HPV prevalence among the participants from different age groups $(P<0.001$, Table 2$)$. Furthermore, agespecific HPV distribution showed a bimodal pattern. Two peaks of HPV infection were detected in the population of the youngest group $(33.11 \%)$ and the oldest group (25.95\%), suggesting young women may be more prone to HPV infection.

The prevalence of HR-HPV only, LR-HPV only, and mixed LR- and HR-HPV infections were $15.17 \%$ (95\% CI, 15.02-15.32\%), $2.07 \%$ (95\% CI, 2.01$2.13 \%$ ), $1.47 \%$ (95\% CI, $1.42-1.52 \%$ ), respectively, and a significant difference was observed in the distribution of HR-HPV only, LR-HPV only, and mixed LR- and HR-HPV infections among the six age groups $(P<0.001$, Table 2$)$. The highest prevalence of HR-HPV only infection (20.04\%) was still found in the youngest age group, and it decreased gradually as the age increased (21-30, 31-40 and 41-50 years) before hitting another increasing trend in the older age groups (51-60 and $>60$ years), showing a bimodal curve. A similar age-specific prevalence pattern was also noted in the LR-HPV only, and mixed LR- and HR-HPV infections. 
Table 1 Overall prevalence of HPV genotype in single and multiple infections

\begin{tabular}{|c|c|c|c|c|c|c|}
\hline \multirow[t]{2}{*}{ Genotypes } & \multicolumn{2}{|c|}{ Single infection } & \multicolumn{2}{|c|}{ Multiple infections } & \multicolumn{2}{|l|}{ Total } \\
\hline & $(n, \%)$ & $95 \% \mathrm{Cl}$ & $(n, \%)$ & $95 \% \mathrm{Cl}$ & $(n, \%)$ & $95 \% \mathrm{Cl}$ \\
\hline \multicolumn{7}{|l|}{ HR-HPV* } \\
\hline 52 & $7457(3.47)$ & $3.39-3.55$ & $3537(1.65)$ & $1.60-1.70$ & $10,994(5.12)$ & $5.03-5.21$ \\
\hline 16 & $4183(1.95)$ & $1.89-2.01$ & $2175(1.01)$ & $0.97-1.05$ & $6358(2.96)$ & $2.89-3.03$ \\
\hline 58 & $3267(1.52)$ & $1.47-1.57$ & $2128(0.99)$ & $0.95-1.03$ & $5395(2.51)$ & $2.44-2.58$ \\
\hline 53 & $2261(1.05)$ & $1.01-1.09$ & $1683(0.78)$ & $0.74-0.82$ & $3944(1.84)$ & $1.78-1.90$ \\
\hline 39 & $1747(0.81)$ & $0.77-0.85$ & $1330(0.62)$ & $0.59-0.65$ & $3077(1.43)$ & $1.38-1.48$ \\
\hline 51 & $1284(0.60)$ & $0.57-0.63$ & $1030(0.48)$ & $0.45-0.51$ & $2314(1.08)$ & $1.04-1.12$ \\
\hline 68 & $1187(0.55)$ & $0.52-0.58$ & $1092(0.51)$ & $0.48-0.54$ & $2279(1.06)$ & $1.02-1.10$ \\
\hline 33 & $1231(0.57)$ & $0.54-0.60$ & $1031(0.48)$ & $0.45-0.51$ & $2262(1.05)$ & $1.01-1.09$ \\
\hline 18 & $1279(0.60)$ & $0.57-0.63$ & $878(0.41)$ & $0.38-0.44$ & $2157(1.00)$ & $0.96-1.04$ \\
\hline 31 & $841(0.39)$ & $0.36-0.42$ & $664(0.31)$ & $0.29-0.33$ & $1505(0.70)$ & $0.67-0.74$ \\
\hline 66 & $598(0.28)$ & $0.26-0.30$ & $639(0.30)$ & $0.28-0.32$ & $1237(0.58)$ & $0.55-0.61$ \\
\hline 56 & $501(0.23)$ & $0.21-0.25$ & $520(0.24)$ & $0.22-0.26$ & $1021(0.48)$ & $0.45-0.51$ \\
\hline 59 & $436(0.20)$ & $0.18-0.22$ & $368(0.17)$ & $0.15-0.19$ & $804(0.37)$ & $0.35-0.40$ \\
\hline 45 & $225(0.10)$ & $0.09-0.11$ & $258(0.10)$ & $0.11-0.14$ & $483(0.22)$ & $0.20-0.24$ \\
\hline 35 & $258(0.12)$ & $0.11-0.14$ & $200(0.09)$ & $0.08-0.10$ & $458(0.21)$ & $0.19-0.23$ \\
\hline \multicolumn{7}{|l|}{ LR- HPV } \\
\hline 81 & $2240(1.04)$ & $1.00-1.08$ & $1743(0.81)$ & $0.77-0.85$ & 3983 (1.86) & $1.80-1.92$ \\
\hline 6 & $765(0.36)$ & $0.34-0.39$ & $723(0.34)$ & $0.32-0.37$ & $1488(0.69)$ & $0.66-0.73$ \\
\hline 11 & $643(0.30)$ & $0.28-0.32$ & $519(0.24)$ & $0.22-0.26$ & $1162(0.54)$ & $0.51-0.57$ \\
\hline 44 & $380(0.18)$ & $0.16-0.20$ & $342(0.16)$ & $0.14-0.18$ & $722(0.34)$ & $0.32-0.37$ \\
\hline 42 & $173(0.08)$ & $0.07-0.09$ & $148(0.07)$ & $0.06-0.08$ & $321(0.15)$ & $0.13-0.17$ \\
\hline 43 & $92(0.04)$ & $0.03-0.05$ & $105(0.05)$ & $0.04-0.06$ & $197(0.09)$ & $0.08-0.10$ \\
\hline Total & $31,048(14.46)$ & $14.31-14.61$ & 9120 (4.25) & $4.17-4.34$ & 40,168 (18.71) & $18.55-18.88$ \\
\hline
\end{tabular}

Single infection versus multiple infections considering HR-HPV ${ }^{*} P<0.001$ and LR- HPV ${ }^{\#} P<0.001$. HPV Human papillomavirus, HR-HPV High-risk HPV, LR-HPV Lowrisk HPV, Cl Confidence interval

\section{Prevalence of single and multiple HPV infections}

The prevalence of single HPV infection was $14.46 \%$ (14.46\%; 95\% CI, 14.31-14.61\%) accounting for $77.30 \%$ of the 40,168 HPV-positive cases, whereas multiple infections (4.25\%; 95\% CI, 4.17-4.34\%) were rare. For those infected with multiple HPV genotypes, the prevalence decreased significantly as the number of infected HPV genotypes increased $(P<$ 0.001, Table 2). Notably, four participants were found to be infected with nine HPV types (data not shown).

In addition, there was a significant difference in the distribution of single and multiple HPV infections among different age groups $(P<0.001$, Table 2$)$. Young women aged less than 21 years had the highest prevalence of both single (19.47\%) and multiple HPV infection (13.64\%), which further confirmed our previous finding [15] of a high susceptibility to HPV infection in the young population.
Prevalence of LR-HPV only, HR-HPV only, and mixed LRand HR-HPV infections by number of HPV genotypes

A significantly different distribution was observed for LR-HPV only, HR-HPV only, and Mixed LR- and HR-HPV infections among women co-infected with a different number of HPV genotypes $(P<0.001)$. Both LR- and HR-HPV only infection had the highest prevalence in single HPV infection (2.00\% vs. $12.46 \%)$, which declined gradually as the number of coinfections increased. Particularly, HR-HPV only infection took up the majority in women with double and triple HPV infection, and mixed LR- and HR-HPV infections correlated with those infected with more than three HPV genotypes, while none of the infections with three or more genotypes were detected in those with LR-HPV only infections (Table 3). These data indicated that LR-HPV only infections primarily occurred in single HPV infections, while mixed HR$\mathrm{HPV}$ infections in multiple HPV infections. 


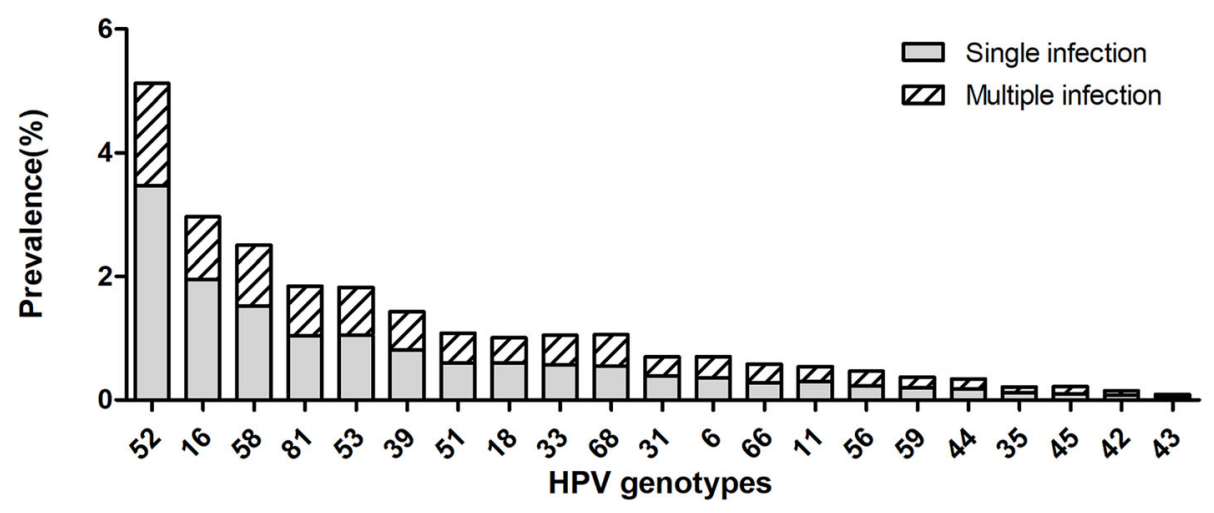

Fig. 1 Prevalence of HPV infections. a: Prevalence of HPV infections among females, 2012-2018; b: genotype-specific distribution of HPV infections, 2012-2018. The six top prevalent HPV genotypes were HPV52 (5.12\%), $-16(2.96 \%),-58(2.51 \%),-53(1.84 \%),-39(1.43 \%)$, and -51 (1.08\%) respectively. HPV genotypes, except for HPV51, - 39 and -53, presented a tendency of decrease from 2012 to 2018

\section{Discussion}

Here we measured the prevalence of 21 distinct HPV types and found the overall prevalence of HPV in this representative sample of women was $18.71 \%$ and HRHPV of $15.17 \%$, amongst 214,715 women prior to HPV vaccination. The high HR-HPV prevalence indicated the inadequacy of routine cervical screening in the region. Additionally, the large sample size allows for precise estimation of both increases and decreases in HPV type specific prevalence, which could be used as a baseline of comparison to future sampling of the entire population.

Global HPV prevalence estimates are known to vary by the region, study design, target population and calendar time [16]. According to the previous reports, HPV positive rates range from 6.70 to $44.50 \%$ in China [11]. Notably, Western Asia (1.70\%) and North America (4.70\%) had a low rates of HPV infection, while East Africa $(33.6 \%)$ and the Caribbean (35.4\%) had a high rates of HPV infection [17-19]. HR-HPV genotype distribution was also heterogeneous, ranging from 9.90-27.50\% in China, which is $15.17 \%$ in current study, as with that in Guangdong (20.02\%), Guiyang (20.45\%) and Nanning (22.28\%) [20]. The variation was expected to be explained by cultural diversity, the sampling strategy, methods and devices, as well as the sensitivity and specificity of the HPV detection assays.

In present study, the sampling methods and devices, and Hybribio test were enrolled for HPV detection assays unaltered for over 7 years, which could monitor the annual changes of HPV prevalence sufficiently. We found HPV prevalence showed a significant downward trend from 2012 to 2016. Analogous results of the HRHPV positive rates were also found to decline from $25.3 \%$ in 2007 to $18.4 \%$ in 2014, in Guangzhou [21]. This decline may be due to the following reasons: 1) emphasis on cervical cancer has led to increase participation in screening, including those without cervical abnormalities; 2) in the last decade, some women tested positive for HPV became negative, due to immunization and treatment; and 3) individuals were more likely to be aware of HPV and HPV vaccination following the continuous improvements of living conditions and public

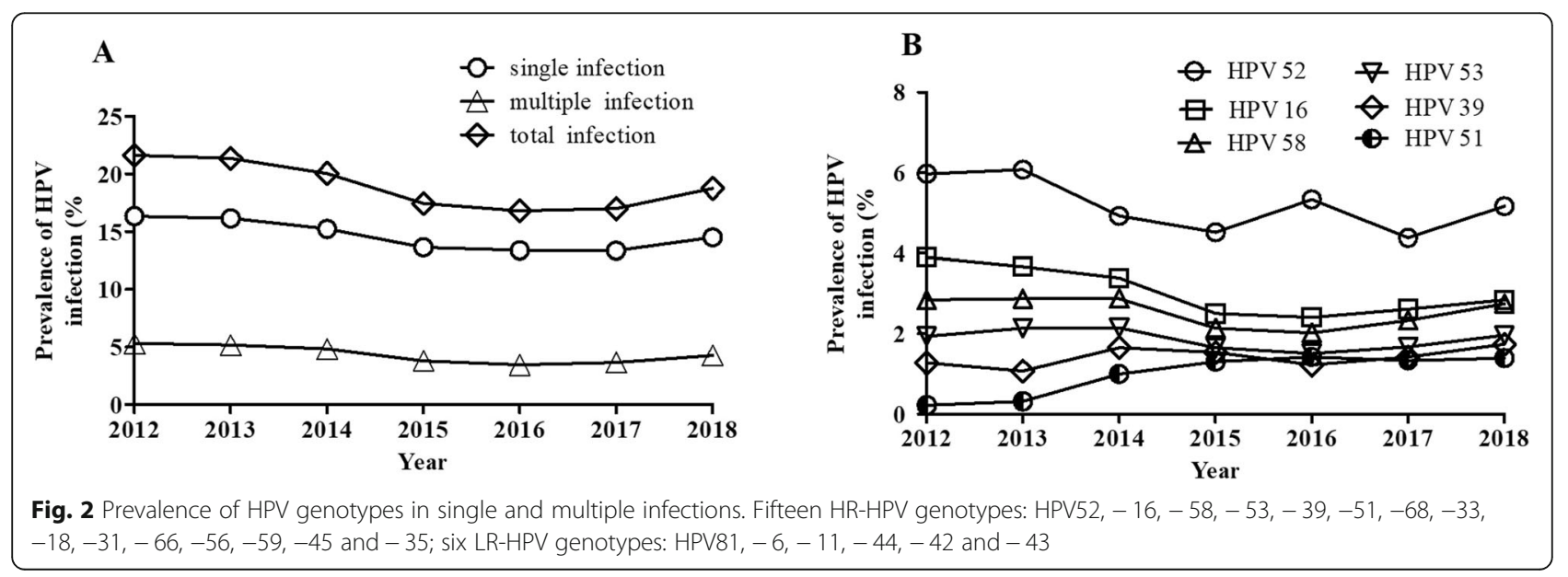


Table 2 Age-specific distribution of HPV infection

\begin{tabular}{|c|c|c|c|c|c|c|c|c|}
\hline & \multicolumn{7}{|c|}{ Age, years (n, \%) } & \multirow[t]{2}{*}{$P$} \\
\hline & $\begin{array}{l}<21 \\
(n=2111)\end{array}$ & $\begin{array}{l}21-30(n=45 \\
211)\end{array}$ & $\begin{array}{l}31-40(n=70 \\
681)\end{array}$ & $\begin{array}{l}41-50(n=68 \\
488)\end{array}$ & $\begin{array}{l}51-60(n=22 \\
142)\end{array}$ & $\begin{array}{l}>60 \\
(n=6082)\end{array}$ & Total & \\
\hline $\begin{array}{l}\text { HPV positive } \\
\text { numbers }\end{array}$ & $699(33.11)$ & $9073(20.07)$ & $12,223(17.29)$ & $11,801(17.23)$ & $4794(21.65)$ & $1578(25.95)$ & $\begin{array}{l}40,168 \\
(18.71)\end{array}$ & $<0.001$ \\
\hline \multicolumn{9}{|l|}{ Number of co-infections } \\
\hline single HPV genotype & $411(19.47)$ & $6818(15.08)$ & $9795(13.86)$ & $9464(13.82)$ & $3519(15.89)$ & $1041(17.12)$ & $\begin{array}{l}31,048 \\
(14.16)\end{array}$ & \multirow[t]{5}{*}{$<0.001$} \\
\hline $\begin{array}{l}\text { double HPV } \\
\text { genotypes }\end{array}$ & $167(7.91)$ & $1671(3.70)$ & $1957(2.77)$ & $1882(2.75)$ & $906(4.09)$ & $343(5.64)$ & $6926(3.23)$ & \\
\hline triple HPV genotypes & $74(3.51)$ & $438(0.97)$ & $349(0.49)$ & $350(0.51)$ & $257(1.16)$ & $121(1.99)$ & $1589(0.74)$ & \\
\hline $\begin{array}{l}\text { Quadruple HPV } \\
\text { genotypes }\end{array}$ & $32(1.52)$ & $108(0.24)$ & $90(0.13)$ & $78(0.11)$ & $74(0.33)$ & $45(0.74)$ & $427(0.20)$ & \\
\hline $\begin{array}{l}\geq \text { five HPV } \\
\text { genotypes }\end{array}$ & $15(0.71)$ & $38(0.08)$ & $32(0.05)$ & $27(0.04)$ & $38(0.17)$ & $28(0.46)$ & $178(0.08)$ & \\
\hline \multicolumn{9}{|l|}{ HPV genotype } \\
\hline HR-HPV only & $423(20.04)$ & $7122(15.75)$ & $10,146(14.35)$ & $9742(14.22)$ & $3881(17.53)$ & $1259(20.70)$ & $\begin{array}{l}32,573 \\
(15.17)\end{array}$ & \multirow[t]{3}{*}{$<0.001$} \\
\hline LR-HPV only & $114(5.40)$ & $1123(2.48)$ & $1309(1.85)$ & $1293(1.89)$ & $484(2.19)$ & $125(2.06)$ & $4448(2.07)$ & \\
\hline $\begin{array}{l}\text { mixed LR- and HR- } \\
\text { HPV }\end{array}$ & $162(7.67)$ & $828(1.83)$ & $768(1.09)$ & $766(1.12)$ & $429(1.94)$ & 194 (3.19) & $3147(1.47)$ & \\
\hline
\end{tabular}

HPV Human papillomavirus, HR-HPV High-risk HPV, LR-HPV, Low-risk HPV, Cl Confidence interval

health awareness. Several studies have demonstrated the association of HPV with economic development. Women from impoverished countries and areas suffered a high prevalence of HPV, e.g., $66.7 \%$ among young females in South Africa and $44.5 \%$ in Henan province of China $[19,22]$. On the contrary, a low rate of $6.7 \%$ was found among women in Beijing-the capital of China, which remained the most flourishing cultural and economic center in China, and also had the excellent healthcare system, indicating a strong correlation of HPV with socioeconomic development [23].

We found HPV52 was the most commonly detected genotype, in agreement with that stated in Japan, Taiwan, and eastern Africa [24]. However, it was inconsistent with the data reported that HPV16 was the predominant genotype in other studies [3] and HPV35 in
sub-Saharan Africa [25]. Moreover, prevalence of both HPV52 (5.12\%) and - 58 (2.51\%) was higher than that reported (2.3 and 1.0\% respectively) in the United States [3], and a nationwide population-based investigation in 37 cities in China [20]. HPV 16 is the strain most likely to cause cancer. Thus, clinic-based studies usually found higher prevalence of HPV16 than population-based studies [24, 26]. We found a relatively low prevalence of HPV16, which was in accordance with an estimated HPV16 prevalence of $3.2 \%$ from a meta-analysis of 1 million women with normal cytology [27]. It is possible that most women seemingly healthy from the physical examination center enrolled in our study were more likely to detect HPV types not related to cervical infection. Our data also indicated that HPV52, - 16, and - 58 were consistently the top three HR-HPV genotypes from

Table 3 Prevalence of LR-HPV only, HR-HPV only, and Mixed LR- and HR-HPV infections in women co-infection with different numbers

\begin{tabular}{|c|c|c|c|c|c|c|c|c|}
\hline \multirow[t]{2}{*}{ Number of co-infections } & \multicolumn{2}{|c|}{ LR-HPV only } & \multicolumn{2}{|l|}{ HR-HPV only } & \multicolumn{2}{|c|}{ mixed LR- and HR-HPV } & \multicolumn{2}{|l|}{ Total } \\
\hline & $\mathrm{n},(\%)$ & $95 \% \mathrm{Cl}$ & $n,(\%)$ & $95 \% \mathrm{Cl}$ & $\mathrm{n},(\%)$ & $95 \% \mathrm{Cl}$ & $n,(\%)$ & $95 \% \mathrm{Cl}$ \\
\hline Total & $4448(2.07)$ & $2.01-2.13$ & $32,571(15.17)$ & $15.02-15.32$ & $3149(1.47)$ & $1.42-1.52$ & $40,168(18.71)$ & $18.55-18.88$ \\
\hline Single HPV genotype & $4293(2.00)$ & $1.94-2.06$ & $26,755(12.46)$ & $12.32-12.60$ & NA & NA & $31,048(14.46)$ & $14.31-14.61$ \\
\hline Double HPV genotypes & $152(0.07)$ & $0.06-0.08$ & $4752(2.21)$ & $2.15-2.27$ & $2022(0.94)$ & $0.90-0.98$ & $6926(3.23)$ & $3.16-3.31$ \\
\hline Triple HPV genotypes & $3(0.00)$ & NA & $849(0.40)$ & $0.37-0.43$ & $737(0.34)$ & $0.32-0.37$ & $1589(0.74)$ & $0.70-0.78$ \\
\hline Quadruple HPV genotypes & $0(0.00)$ & NA & $169(0.08)$ & $0.07-0.09$ & $258(0.12)$ & $0.11-0.14$ & $427(0.20)$ & $0.18-0.22$ \\
\hline ZFive HPV genotypes & $0(0.00)$ & NA & $46(0.02)$ & $0.01-0.03$ & $132(0.06)$ & $0.05-0.07$ & $178(0.08)$ & $0.07-0.09$ \\
\hline$P$ & $<0.001$ & & $<0.001$ & & $<0.001$ & & $<0.001$ & \\
\hline
\end{tabular}


2012 to 2018, suggesting the HPV vaccine covering these HR-HPV types is routinely recommended, especially for those females at young ages exposed to HPV in this region.

Evaluations in the United State showed that young women had the highest HPV prevalence [3], consistent with our the findings of the highest HPV prevalence (33.11\%) among women aged less than 21 years. Young women often have a high infection rate, mainly because they are sexually more active before their immune systems become less sensitive [17]. Although they were known to have high risks for HPV infection, however it is temporary and supposed to disappear within a year or two, and thus its prevalence declined gradually with respect to ages [18]. Predictably, the prevalence of HPV slightly declined in middle ages, yet significantly increased among the oldest people, which are consistent with those in most developed countries and the data from Bruni and colleagues [27]. The mechanism of this increase in infection rates is unclear at present. Other than persistent infections that seem to be more prominent among females at older ages [19], this increase could be also explained by re-marriage, reactivation of latent HPV in menopausal women and the cohort variation [16]. In addition, women aged 31-40 years were the most common population for screening, and those aged 41-50 years showed an increase trend in this study, thus routine screening is strongly recommended for women over 30 .

In an unweighted analysis of women with multiple HPV genotype infections, we found HR-HPV genotypes accounted for over $80 \%$ of multiple infections, which mainly occurred at ages either younger than 21 or older than 60. Some have suggested HPV infection with multiple genotypes may prolong the duration of infection and increase the risk of cervical cancer and cervical precancerous lesions [28], leading to a complication of multiple infections among older women. Multiple infections were believed to have competitive and/or cooperative interactions between HPV genotypes [29]. Although the mechanisms and potential oncogenic effects of multiple genotype infections still require further investigation, this study could still be beneficial to the development of HPV prophylactic vaccines.

The overall strengths of this study include the large sample size, unaltered HPV genotyping methods over 7 years, decade-long study period measuring the trends of HPV infection on a yearly basis, and the use of PCR testing rather than serologic tests, which allows for accurate determination of simultaneous co-infection, all of that provide a good baseline for epidemiological surveillance after the introduction of HPV vaccine at population level. However, several limitations exist in our study. Firstly, HPV DNA testing does not reflect the previous and cumulative incidence of HPV infections, but only the current infection status. Secondly, our study included specimens from women, without pathological data, such as, cervical cytology and histology results, which was unable to explain the relationship between HPV infection and pathology. Thirdly, no male samples were analyzed, so our study would not be able to represent the infection of HPV in general population in the region. Lastly, the detailed information about the patients, such as education level, economic status and background related to HPV infection, were not documented in this study, hindering a more comprehensive evaluation of the effects of these factors on the prevalence of HPV infection.

\section{Conclusions}

In conclusion, our study provides an estimate of HPV infection among a large female population in Southern China. Overall, HPV prevalence was high (18.71\%), showing a downward trend from 2012 to 2018, and women aged less than 21 years old showed the highest HPV prevalence. Importantly, HPV52, - 16, and - 58 were the major genotypes at all times, regardless of the fact that their ranks varied with respect to the ages and years. Our study enables the estimations of HPV vaccine impact among women spanning a broad age range, provide guidance for clinical care and public health policy including cervical screening and vaccination, and would also be useful for the other low- and middle-income areas with a heavy HPV infection burden to fight against cervical cancer.

\section{Abbreviations \\ HPV: Human papillomavirus; HR-HPV: High-risk HPV; LR-HPV: Low- risk HPV; $\mathrm{Cl}$ : Confidence interval}

\section{Acknowledgements}

The authors would like to thank all the participants, as well as investigators for their contribution to the study. We also thank Dr. Chenxu Guo (The Johns Hopkins University, School of Medicine) for the careful reading of the manuscript and Jian Zhang (Chenzhou NO.1 People's Hospital) for assistance with statistical analysis.

\section{Authors' contributions}

$L-P L, Z-Y L$ and $H-L C$ drafted the manuscript and participated in the design of the study. L-PL, H-LC, Z-YL and X-GD performed the statistical analysis. PH, QTL, Y-HJ, Y-NZ, Q-ZL, QL, S-TL, FY and HL carried out the sample collection and HPV detection. All authors made substantive intellectual contributions, performed research, and read and approved the final manuscript.

\section{Funding}

This work was supported by Hunan Health Commission project: Prevalence of Chlamydia trachomatis and HPV in Southern China (B2019002) and Chenzhou Science and technology Bureau: Associations of Chlamydia trachomatis and HPV Co-infection with cervical neoplasia (yfzx201908, zdyf201941 and zdyf201947). The funder had no role in the study design, data collection and analysis, interpretation of data, and writing the manuscript. 


\section{Availability of data and materials}

The datasets used and/or analysed during the current study are available from the corresponding author on reasonable request.

\section{Declarations}

\section{Ethics approval and consent to participate}

The study was approved by the ethics Committee of Chenzhou NO.1 People's Hospital. No informed consent or other action on the part of the patients was required owing to anonymous analyses of the data.

\section{Consent for publication}

Not applicable.

\section{Competing interests}

The authors declare that they have no competing interests.

\section{Author details}

TThe First School of Clinical Medicine, Southern Medical University, Chenzhou NO.1 People's Hospital, Chenzhou 423000, P.R. China. ${ }^{2}$ Institute of Pathogenic Biology, Hengyang Medical College, Hunan Provincial Key Laboratory for Special Pathogens Prevention and Control, Hunan Province Cooperative Innovation Center for Molecular Target New Drug Study, University of South China, Hengyang 421001, P.R. China.

\section{Received: 31 March 2020 Accepted: 28 March 2021}

\section{Published online: 07 April 2021}

\section{References}

1. Senkomago V, Henley SJ, Thomas CC, Mix JM, Markowitz LE, Saraiya M. Human papillomavirus-attributable cancers - United States, 2012-2016. MMWR Morb Mortal Wkly Rep. 2019;68(33):724-8. https://doi.org/10.15585/ mmwr.mm6833a3.

2. Siegel RL, Miller KD, Jemal A. Cancer statistics, 2015. CA Cancer J Clin. 2015; 65(1):5-29. https://doi.org/10.3322/caac.21254.

3. Dunne EF, Unger ER, Sternberg M, McQuillan G, Swan DC, Patel SS, et al. Prevalence of HPV infection among females in the United States. JAMA. 2007;297(8):813-9. https://doi.org/10.1001/jama.297.8.813.

4. Chen $W$, Zheng $R$, Zeng $H$, Zhang $S$. The incidence and mortality of major cancers in China, 2012. Chin J Cancer. 2016;35(1):73. https://doi.org/10.1186/ s40880-016-0137-8

5. Oliver SE, Unger ER, Lewis R, McDaniel D, Gargano JW, Steinau M, et al. Prevalence of human papillomavirus among females after vaccine introduction-National Health and nutrition examination survey, United States, 2003-2014. J Infect Dis. 2017;216(5):594-603. https://doi.org/10.1093/ infdis/jix244

6. Bellaminutti S, Seraceni S, De Seta F, Gheit T, Tommasino M, Comar M. HPV and chlamydia trachomatis co-detection in young asymptomatic women from high incidence area for cervical cancer. J Med Virol. 2014;86(11):19205. https://doi.org/10.1002/jmv.24041.

7. Aro K, Nieminen P, Louvanto K, Jakobsson M, Virtanen S, Lehtinen M, et al. Age-specific HPV type distribution in high-grade cervical disease in screened and unvaccinated women. Gynecol Oncol. 2019;154(2):354-9. https://doi.org/10.1016/j.ygyno.2019.05.024.

8. Lee NR, Winer RL, Cherne S, Noonan CJ, Nelson L, Gonzales AA, et al. Human papillomavirus prevalence among American Indian women of the Great Plains. J Infect Dis. 2019;219(6):908-15. https://doi.org/10.1093/infdis/ jiy600.

9. Wang Y, Meng Y, Li W, Zhang X, Deng Z, Hu M, et al. Prevalence and characteristics of hrHPV infection among 414,540 women: a multicenter study in central and eastern China. J Cancer. 2019;10(8):1902-8. https://doi. org/10.7150/jca.30157.

10. Ferre VM, Ekouevi DK, Gbeasor-Komlanvi FA, Collin G, Le Hingrat Q, Tchounga B, et al. Prevalence of human papillomavirus, human immunodeficiency virus and other sexually transmitted infections among female sex workers in Togo: a national cross-sectional survey. Clin Microbiol Infect. 2019;25(12):1560 e1561-7.

11. Li K, Li Q, Song L, Wang D, Yin R. The distribution and prevalence of human papillomavirus in women in mainland China. Cancer. 2019;125(7):1030-7. https://doi.org/10.1002/cncr.32003.
12. Owusu-Edusei K Jr, Chesson HW, Gift TL, Tao G, Mahajan R, Ocfemia MC, et al. The estimated direct medical cost of selected sexually transmitted infections in the United States, 2008. Sex Transm Dis. 2013;40(3):197-201. https://doi.org/10.1097/OLQ.0b013e318285c6d2.

13. Chow EP, Danielewski JA, Fehler G, Tabrizi SN, Law MG, Bradshaw CS, et al. Human papillomavirus in young women with chlamydia trachomatis infection 7 years after the Australian human papillomavirus vaccination programme: a cross-sectional study. Lancet Infect Dis. 2015;15(11):1314-23. https://doi.org/10.1016/S1473-3099(15)00055-9.

14. Braaten KP, Laufer MR. Human papillomavirus (HPV), HPV-related disease, and the HPV vaccine. Rev Obstet Gynecol. 2008;1(1):2-10.

15. Chen H, Luo L, Wen $Y$, He B, Ling H, Shui J, et al. Chlamydia trachomatis and human papillomavirus infection in women from southern Hunan Province in China: a large observational study. Front Microbiol. 2020;11:827. https://doi.org/10.3389/fmicb.2020.00827.

16. Brotherton JM, Hawkes D, Sultana F, Malloy MJ, Machalek DA, Smith MA, et al. Age-specific HPV prevalence among 116,052 women in Australia's renewed cervical screening program: a new tool for monitoring vaccine impact. Vaccine. 2019;37(3):412-6. https://doi.org/10.1016/j.vaccine.2018.11.075.

17. Jiang L, Tian X, Peng D, Zhang L, Xie F, Bi C, et al. HPV prevalence and genotype distribution among women in Shandong Province, China: analysis of 94,489 HPV genotyping results from Shandong's largest independent pathology laboratory. PLoS One. 2019;14(1):e0210311. https:/doi.org/10.1371/journal.pone.0210311.

18. Wang J, Tang D, Wang K, Wang J, Zhang Z, Chen Y, et al. HPV genotype prevalence and distribution during 2009-2018 in Xinjiang, China: baseline surveys prior to mass HPV vaccination. BMC Womens Health. 2019;19(1):90. https://doi.org/10.1186/s12905-019-0785-3.

19. Zhao R, Zhang WY, Wu MH, Zhang SW, Pan J, Zhu L, et al. Human papillomavirus infection in Beijing, People's Republic of China: a population-based study. Br J Cancer. 2009;101(9):1635-40. https://doi.org/10.1038/sj.bjc.6605351.

20. Wang R, Guo XL, Wisman GB, Schuuring E, Wang WF, Zeng ZY, et al. Nationwide prevalence of human papillomavirus infection and viral genotype distribution in 37 cities in China. BMC Infect Dis. 2015;15(1):257. https://doi.org/10.1186/s12879-015-0998-5.

21. Chen $X, X u H, X u$ W, Zeng W, Liu J, Wu Q, et al. Prevalence and genotype distribution of human papillomavirus in 961,029 screening tests in southeastern China (Zhejiang Province) between 2011 and 2015. Sci Rep. 2017;7(1):14813. https://doi.org/10.1038/s41598-017-13299-y.

22. Mbulawa ZZA, van Schalkwyk C, Hu NC, Meiring TL, Barnabas S, Dabee S, et al. High human papillomavirus (HPV) prevalence in south African adolescents and young women encourages expanded HPV vaccination campaigns. PLoS One. 2018;13(1):e0190166. https://doi.org/10.1371/journal.pone.0190166.

23. Thompson EL, Wheldon CW, Rosen BL, Maness SB, Kasting ML, Massey PM. Awareness and knowledge of HPV and HPV vaccination among adults ages 27-45 years. Vaccine. 2020;38(15):3143-8. https:/doi.org/10.1016/j.vaccine.2020.01.053.

24. de Sanjose S, Diaz M, Castellsague X, Clifford G, Bruni L, Munoz N, et al. Worldwide prevalence and genotype distribution of cervical human papillomavirus DNA in women with normal cytology: a meta-analysis. Lancet Infect Dis. 2007;7(7):453-9. https://doi.org/10.1016/S1473-3099(07)70158-5.

25. Torres-Poveda K, Ruiz-Fraga I, Madrid-Marina V, Chavez M, Richardson V. High risk HPV infection prevalence and associated cofactors: a population-based study in female ISSS TE beneficiaries attending the HPV screening and early detection of cervical cancer program. BMC Cancer. 2019;19(1):1205. https:/doi.org/10.1186/s12885-019-6388-4.

26. Castle PE, Schiffman M, Bratti MC, Hildesheim A, Herrero R, Hutchinson ML, et al. A population-based study of vaginal human papillomavirus infection in hysterectomized women. J Infect Dis. 2004;190(3):458-67. https//doi.org/10.1086/421916.

27. Bruni L, Diaz M, Castellsague X, Ferrer E, Bosch FX, de Sanjose S. Cenvical human papillomavirus prevalence in 5 continents: meta-analysis of 1 million women with normal cytological findings. J Infect Dis. 2010;202(12):1789-99. https//doi.org/10.1086/657321.

28. Schmitt M, Depuydt C, Benoy I, Bogers J, Antoine J, Arbyn M, et al. Multiple human papillomavirus infections with high viral loads are associated with cervical lesions but do not differentiate grades of cervical abnormalities. J Clin Microbiol. 2013;51(5):1458-64. https://doi.org/10.1128/JCM.00087-13.

29. Senapati R, Nayak B, Kar SK, Dwibedi B. HPV genotypes co-infections associated with cervical carcinoma: special focus on phylogenetically related and non-vaccine targeted genotypes. PLoS One. 2017;12(11):e0187844. https://doi.org/10.1371/journal.pone.0187844.

\section{Publisher's Note}

Springer Nature remains neutral with regard to jurisdictional claims in published maps and institutional affiliations. 